\title{
Health-related quality of life and life satisfaction in patients following surgically treated pelvic ring fractures. A prospective observational study with two years follow-up
}

\author{
Tomas Borg ${ }^{a, *}$, Per Berg ${ }^{a}$, Kerstin Fugl-Meyer ${ }^{b}$, Sune Larsson $^{a}$ \\ ${ }^{a}$ Department of Orthopaedic Surgery, Uppsala University Hospital, Uppsala, Sweden \\ ${ }^{\mathrm{b}}$ Center for Andrology and Sexual Medicine, Karolinska University Hospital, Stockholm, Sweden
}

\section{A R T I C L E I N F O}

Article history:

Accepted 18 November 2009

\section{Keywords:}

Pelvic

Fractures

Surgery

Treatment

Quality of life

SF-36

Li-Sat11

\begin{abstract}
A B S T R A C T
Background: Pelvic ring fractures caused by high-energy trauma are severe injuries with well described radiological and clinical outcomes, whereas description from the patient's perspective is less well documented. The purpose of this study was to investigate patient-reported outcome following surgical treatment of pelvic fractures using quality of life instruments.

Methods: All 54 patients (28 male/26 female, ages 16-68) with pelvic fractures referred to our institution for surgical treatment 2003-2005 were prospectively included. The most common trauma was motor vehicle accident (44\%). Additional injuries were seen in $74 \%$ and in $31 \%$ the ISS was $\geq 16$. There were $31 \mathrm{~B}$ and $23 \mathrm{C}$ type fractures. Patients were followed for two years using two validated questionnaires, SF-36 and LiSat-11, the latter an instrument consisting of 11 questions for evaluation of satisfaction with different aspects of life.

Results: 45 patients could be followed according to the study protocol for two years while 2 were untraceable and 1 died from unrelated causes. Of 6 nonresponders, 5 were unable due to psychiatric disorder. At two years pelvic fracture patients scored lower than the reference population in both physical and mental domains (SF-36). Highest mean score, 68, was in the domain Social Function (norm 89) while lowest mean score, 38 , was in the domain Role Physical (norm 86). The mean score closest to the normative was for general health with 61 for patients and 78 for the normative group. In LiSat-11 pelvic fracture patients scored lower than the reference population in all areas. Satisfaction with life as a whole was $31 \%$ compared with $60 \%$ in the normative group.

Conclusions: Two years after surgical treatment of pelvic ring fractures, patients reported substantially lower quality of life for both physical and mental domains, when compared with a reference population, even when radiological and clinical outcomes were considered favourable.
\end{abstract}

(c) 2009 Elsevier Ltd. All rights reserved.

\section{Introduction}

Pelvic ring fractures and disruptions requiring surgery are severe injuries often caused by high-energy trauma, and frequently associated with other injuries. There is considerable morbidity not only due to the pelvic injury itself but also due to, for instance, associated vascular, neurological and urological injuries..$^{2,7,22}$ Common indications for surgery are instability and/or displacement, with the goal being restoration of stability within an anatomic or near anatomic position of the pelvic ring. There are numerous reports in the literature describing radiological and clinical results. ${ }^{8-10,12,17}$ For instance, it has been shown that an adequate reduction of any posterior displacement is associated

\footnotetext{
* Corresponding author at: Department of Orthopaedic Surgery, Uppsala University Hospital, S-75185 Uppsala, Sweden. Tel.: +46 186110000.

E-mail address: tomas.borg@surgsci.uu.se (T. Borg).
}

with less pain compared with pelvic fractures or disruptions with persistent malreduction of the posterior part, leading to a malunion. ${ }^{14}$

To get a better global understanding of the outcome following various injuries and diseases, studies including patient-reported outcome have in recent years provided new and valuable insights. For patients treated with surgery due to pelvic fractures, however, there is only very limited information available on the outcome using patient-reported outcome instruments. ${ }^{6,14}$ This is despite the fact that pelvic fractures are usually severe injuries, often in younger people, and commonly associated with other injuries. With this in mind there is reason to believe that these injuries might have long-term consequences on general health-related quality of life.

The purpose of this study was to prospectively evaluate patients, surgically treated for acute pelvic fractures and disruptions, using general health-related quality of life instruments, i.e. patient-reported outcome. 


\section{Patients and methods}

\section{Study design}

All patients with pelvic fractures referred to Uppsala University Hospital, Sweden, for surgical treatment 2003-2005 were prospectively included. The study was approved by the research ethics board and patients gave informed consent for their participation. Patients younger than 16 years of age were excluded. In total thirty hospitals referred patients after providing primary care. All patients were followed after surgery prospectively for two years. At two years they were asked to report outcome using two healthrelated quality of life instruments, SF-36 and Lisat-11, and in addition conventional radiographs were obtained and assessed by one observer who had not been part of their treatment.

\section{Patients and treatment}

Patients were operated at our department 1-21 (median 6) days after the trauma by one or two out of three surgeons. Delay more than five days was usually due to concomitant injuries. Indications for surgery included instability and/or displacement exceeding $5 \mathrm{~mm}$.

There were 54 patients, all with closed fractures or disruptions. The gender distribution was 28 males and 26 females, with a mean age of 34 (range 16-68). Only one patient was older than 65 . The most common trauma mechanism was a motor vehicle accident in 21 patients (39\%), followed by jump from height in 12 (22\%) or fall in 7 patients (13\%). One or several additional injuries were seen in $40(74 \%)$ of the patients with the most common location being a lower extremity fracture (Table 1 ). Seventeen patients (31\%) had sustained a severe polytrauma corresponding to an ISS $\geq 16$. The surgical approach was open in 20, percutaneous in 14 and combined in 20 patients. One reoperation was performed due to inadequate reduction based on the postoperative radiographs. Low-molecular weight heparin was used in all patients as prophylaxis against venous thrombosis for at least one week after surgery or longer based on level of mobilisation. Antibiotics, usually cloxacillin, were given perioperatively for prophylaxis against infection.

\section{Radiology}

Diagnostic conventional radiographs and a trauma CT scan with $3 \mathrm{~mm}$ slice thickness was done at the referring hospitals, as part of their trauma imaging protocol. In addition a new CT scan with $1 \mathrm{~mm}$ cuts and 3-D reconstructions were made in almost all cases at arrival to our institution as part of the preoperative planning. Postoperative conventional radiographs included AP pelvis and inlet-outlet views. All the radiological assessment, including classification according to AO/OTA ${ }^{11}$ as well as the assessment on the postoperative and follow-up radiographs was done by one of the authors who was not otherwise involved in the treatment of the patients.

The most common fracture types were B2 and C1 (Table 2). Reduction was assessed on AP and inlet-outlet views and measured according to the German pelvic study group. ${ }^{15}$ Reduc-

Table 1

Anatomical location of associated injuries.

\begin{tabular}{lr}
\hline Head & 7 \\
Chest & 27 \\
Abdomen & 15 \\
Extremities & \\
Upper & 14 \\
Lower & 40 \\
\hline
\end{tabular}

Table 2

OTA classification in pelvic fractures.

\begin{tabular}{lc}
\hline Fracture type & Number \\
\hline B1 & 9 \\
B2 & 14 \\
B3 & 8 \\
C1 & 12 \\
C2 & 7 \\
C3 & 4 \\
Total & 54 \\
\hline
\end{tabular}

tion of the posterior components was anatomic or within $5 \mathrm{~mm}$ residual displacement in 46 patients and more than $5 \mathrm{~mm}$ in 8 patients when assessed on the postoperative radiographs. Thirtyone patients presented with anterior displacement involving the symphysis. Reduction of the symphysis was $\leq 5 \mathrm{~mm}$ in 23 patients, with residual displacement of the symphysis $6-10 \mathrm{~mm}$ in 6 patients and $>10 \mathrm{~mm}$ in 2 patients according to postoperative radiographs. In cases where the posterior stability was considered adequate following posterior fixation a limited anterior displacement of rami fractures was accepted. Thirty-eight patients presented with rami fractures. Postoperative radiographs showed 27 patients with residual displacement of the rami fractures of $<10 \mathrm{~mm}$ and in 11 patients $\geq 10 \mathrm{~mm}$ residual displacement. Two years after surgery final conventional radiographs including AP and inlet-outlet views were taken at the patient's local hospital. The images were digitally transferred to our unit for assessment, done according to the same protocol for all images. In addition, all medical records from referring hospitals were collected and scrutinised for any possible adverse events and complications that might have occurred during the follow-up period without being reported to our unit.

\section{Patient-reported outcomes}

Patients were followed using two validated questionnaires, SF36 and LiSat-11. The two instruments were chosen as they were considered complementary to each other in areas of specific interest following pelvic injuries. SF-36 is a well-known, widely used and validated generic health outcome that consists of eight dimensions. Higher scores are associated with better quality of life and state of health. Age- and gender-matched normative data from Sweden were used as references. ${ }^{19}$ LiSat- 11 is a one-page generic 11 items questionnaire on life satisfaction. The first item characterises satisfaction with "life as a whole". The remaining items characterise satisfaction with "ADL-capacity", "physical health", "psychological health", "sexual life", "partner relationship", "family life", "leisure", "friends and acquaintances", "work" and "financial situation". Each item has six-graded answering alternatives: 1-very dissatisfied/2-dissatisfied/3-rather dissatisfied/4-rather satisfied/5-satisfied/6-very satisfied. The instrument has been validated in a representative sample ( $n$ 2218) of Swedish men and women aged $18-74$ years. It has adequate testretest reliability, discriminate and specificity validities. ${ }^{5}$ The scale can validly be dichotomised into being satisfied (answer alternatives 5 or 6 ) versus not being satisfied (answer alternatives 1-4), where being satisfied indicates that the individual is well adapted and has little or no gap between aspirations and goal achievement. In contrast, being not satisfied means that the individual experiences an aspiration-achievement gap. ${ }^{3}$ For the present investigation a reference sample of those 1898 individuals (1014 men, 884 women) ages $18-74$, who had perceived their health as good, with no history of long lasting (more than one month) disease/disability restricting their life situation and had not been 
medically/surgically treated during the preceding 12 months was selected as a comparator sample. The vast majority had an ongoing partner relationship.

\section{Statistics}

The independent samples $t$-test was used to determine if there were significant differences between the study group and the reference population. Nonparametric methods were used when assumptions for parametric methods were not met. A $p$-value $<0.05$ was considered to be statistically significant. Analysis was performed by an independent biostatistician not directly involved in the project.

\section{Results}

Postoperative complications included one deep vein thrombosis and no pulmonary emboli. There were two deep infections. In one female patient with a C-injury and major vaginal rupture an abscess developed and in one male patient the superficial iliac wing was infected. Both cases were successfully treated with debridement and systemic antibiotics.

\section{Radiology}

All fractures healed within three months. All fractures remained in position except in one patient with a C-injury where there was cranial displacement. All hardware remained in position except in one patient where one of the two SI-screws backed out a few millimetres. For two patients radiographs were not completed at two years for unknown reasons, and another two patients denied radiographs to be taken, in one case due to pregnancy.

\section{Patient-reported outcomes}

45 patients answered the final questionnaire at two years. Two patients were untraceable and 1 had died from an unrelated cause. Out of 6 nonresponders, 5 were unable to respond due to psychiatric disorder. Only one patient with no psychiatric disorder chose not to respond.

\section{SF-36}

At two years fracture patients scored significantly lower than the reference population in all eight domains of SF-36 (Fig. 1). The highest mean score, 68, was in the domain Social Function (norm 89) and the lowest mean score, 38, was in the domain Role Physical (norm 86). The mean score in the domain general health was closest to the norm with 61 for the fracture patients and 78 for the norm reference group. There was a significant difference in SF-36 outcome for fracture type B versus fracture type C, although only

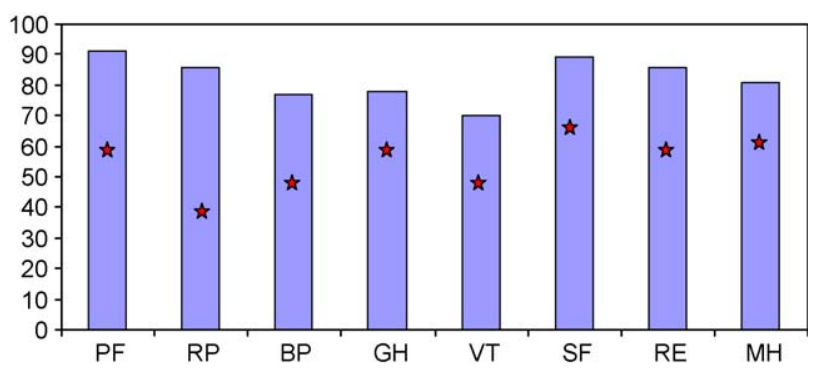

Fig. 1. SF-36 profile in 54 pelvic fracture patients at two years represented by stars, and Swedish norm age- and gender-adjusted represented by bars. PF-Physical functioning, $\mathrm{RP}-$ role limitations due to physical function, $\mathrm{BP}$-bodily pain, $\mathrm{GH}-$ general health, VT-vitality, SF-social functioning, RE-role limitations due to emotional problems, $\mathrm{MH}-$ mental health.
Table 3

Comparison of life satisfaction at two years with normative data.

\begin{tabular}{|c|c|c|c|c|}
\hline \multirow{3}{*}{$\begin{array}{l}\text { LiSat-11 } \\
\text { item }\end{array}$} & \multicolumn{2}{|c|}{ Pelvic fractures $(N=45)$} & \multirow{3}{*}{$\begin{array}{l}\text { Normative } \\
(N=1898) \\
\text { Unsatisfied } \\
\%\end{array}$} & \multirow{3}{*}{$\begin{array}{l}\text { mean } \\
\text { Satisfied } \\
\%\end{array}$} \\
\hline & Unsatisfied & Satisfied & & \\
\hline & $\%$ & $\%$ & & \\
\hline Life as a whole & 55 & $45^{*}$ & 23 & 77 \\
\hline ADL & 43 & $57^{*}$ & 14 & 86 \\
\hline Physical health & 76 & $24^{*}$ & 9 & 91 \\
\hline Psychological health & 61 & $39^{*}$ & 12 & 88 \\
\hline Sexual life & 68 & $32^{*}$ & 42 & 58 \\
\hline Partner relationship & 37 & $63^{*}$ & 19 & 81 \\
\hline Family life & 29 & $71^{*}$ & 15 & 85 \\
\hline Leisure & 67 & $33^{*}$ & 38 & 62 \\
\hline Friends/acquaintances & 52 & $48^{*}$ & 32 & 68 \\
\hline Work & 64 & $36^{*}$ & 42 & 58 \\
\hline Financial situation & 72 & $28^{*}$ & 56 & 44 \\
\hline
\end{tabular}

* Significantly lower than normative with $p<0.05$.

for the domain general health, with fracture type $C$ patients having lower values. For the other seven domains we did not find any significant difference between fracture types with respect to SF-36 outcome.

Summary scores PCS and MCS were not used since several patients with high scores in physical domains had low scores in mental domains, and vice versa. According to the instruction manual for SF- $36^{21}$ such an outcome might give erroneous summation effects, the recommendation is therefore not to use the summary scores in these situations.

\section{LiSat-11}

Patients scored significantly lower than the reference population in all eleven items at two years. The dichotomy of satisfied or very satisfied patients was lower than normative for all items (Table 3). The highest proportion of satisfied patients was in the item "family life" and the worst outcome was in the item "physical health", while the result closest to the normative group was in the item "financial situation". Satisfaction with "life as a whole" showed similar distribution between the fracture patients and the normative group although in general the patients scored lower (Fig. 2). Internal correlation within the LiSat-11 items showed that satisfaction with "life as a whole" correlated to all other 10 items. We did not find any significant difference between fracture type and any of the LiSat-11 items. Satisfaction with "life as a whole" correlated to seven of the eight SF-36 domains, all except Role Physical (Spearman rho 0.05 level, 2-tailed).

We found no significant difference when comparing between the group of patients with or without associated injuries regarding any of the QoL-parameters in both used instruments. We also found no significant difference when comparing between the

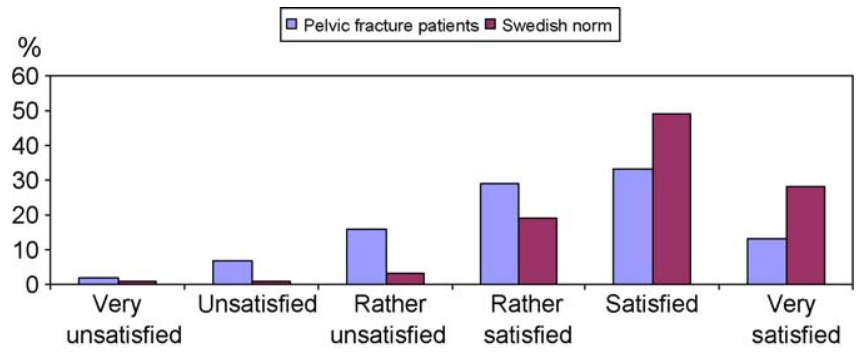

Fig. 2. Satisfaction with "Life as a whole" at two years in 54 pelvic fracture patients described by LiSat-11. 
group of patients with adequate or poor posterior reduction in any of the QoL-measurements.

\section{Discussion}

The overall finding in the present study was the lower values compared to a reference population in almost all aspects of healthrelated quality of life and life satisfaction, differences that remained even at two years following a high-energy pelvic fracture.

The present study has got several strong components where the prospective design is an obvious strength when assessing health-related quality of life variables. Another strength was that the patient series represented a true consecutive series with no selection. All patients referred to us for surgical treatment of a pelvic injury were included. The Swedish system with a personal life-long identification number that makes it possible to trace patients is very beneficial, especially in a study like this with patients living in different parts of the country. With this system, combined with online access to the citizens current addresses, it was possible to trace patients even following relocation and thereby keeping the number of untraceable patients at a very low level compared with the situation in many other countries. For instance, Oliver et al. ${ }^{14}$ described in their study of 55 pelvic fracture patients problems in locating the mobile US population, especially when dealing with a young cohort. They ended up with $35 / 55(64 \%)$ responders. In our study only $2 / 54$ patients (3\%) could not be traced and the response rate was $45 / 54$ (83\%). Noteworthy is that only one of the reachable patients with no psychiatric disorder chose not to respond. In other words, as long as we could trace the patients and they were not suffering from a severe psychiatric disorder that made them unable to respond, patients complied and filled out the questionnaires used. Needless to say, the higher the response rate the more valid conclusions can be made. An additional strength with this study was the actual study design combining patient-reported outcome with the radiographic finding. Access to this combination of subjective and objective data made it possible to assess if specific fracture types and radiological outcome could be associated with a better or worse outcome from the patients perspective.

The limitations for the study include that the pelvic fracture patients, including a subgroup that suffered their injury following a self-destructive jump, could have had a different quality of life (QoL) assessment already before the injury when compared with the reference population. If so, the observed differences between the patients and the normative groups were not fully attributable to the pelvic injury. As always with studies following injuries the QoL prior to the injury was unknown. In some studies including less severe injuries, attempts have been made to ask the patients to retrospectively assess their QoL prior to the injury. In the present study with patients being severely injured we decided not to include such a retrospective assessment due to a potential risk for a systemic over- or under-estimation from the patients when assessing their preinjury QoL. Another limitation was the relatively small number of patients that made it difficult to compare the outcome for different fracture types. We would have liked to perform an analysis of the six different fracture types with regard to quality of life but the subgroups within fracture type B and C were too small for valid interpretation. In fact, even when comparing the major fracture groups, i.e. groups B and C, the limited number of patients made a comparison between groups uncertain. The finding that there was no difference between groups in seven of eight domains is therefore difficult to assess, as well as the finding in one domain "general health" that C fracture patients reported lower values.
As in the present study pelvic fractures are often associated with other major injuries resulting in possible persistent disability. In a review article ${ }^{4}$ it was pointed out that in pelvic fracture patients the rate having associated injuries is very high. When using generic QoL instruments in patients with pelvic fractures and associated injuries, i.e. multiple injured patients, it is obviously not possible to define to what extent a lowered QoL is attributable to the pelvic ring injury and to the associated injuries, respectively. In a study by Michaels et al. ${ }^{13}$ on 165 patients with various injuries following blunt trauma, 51\% were followed for 12 months. Within their study they showed that patients with orthopaedic injuries scored worse than patients without orthopaedic injuries in six of eight SF-36 domains. They concluded that patients with orthopaedic injuries experience prolonged disability, which by one year encompassed physical, psychological, social, occupational and financial components measured with the Sickness Impact Profile work and interviews. This is in agreement with our findings with a wide range of problems reported by patients following pelvic fractures. In a study by Ponsford et al. ${ }^{16}$ on various severe orthopaedic trauma patients, 113 patients out of 342 eligible consenting participants, were recruited during rehabilitation and followed for two years. Their results indicated the presence of very significant levels of disability in all domains of the SF-36 at one and two years in their patients, $93 \%$ of whom were injured in motor vehicle accidents. This is in agreement with our finding with lowered scores in all SF-36 domains at two years following severe orthopaedic trauma in the form of pelvic fractures. In their study, injury severity and type did not predict the patient-reported outcome, although those with lower limb fractures had more pain and poorer physical outcome than patients with fractures in other locations. In our study, apart from having a severe pelvic fracture, a lower extremity fracture was the most common associated injury.

SF-36 has not been used to a large extent in pelvic fracture patients. But in the study by Oliver et al., ${ }^{14}$ a case series consisting of pelvic fracture patients were followed for 16-28 months. Using the SF-36 administered as a postal questionnaire, they showed that in 35 responders out of 55 eligible patients, there was $14 \%$ impairment in physical outcome and 5.5\% impairment in mental outcome score when compared with the normal US population. In our study, at two years after surgical treatment patients reported substantially lowered quality of life for both physical and mental domains compared to an age- and gender-matched reference population. In a study by Van den Bosch et al..$^{20}$ they retrospectively reviewed patients with unstable pelvic ring injuries operated with internal fixation during a seven year period. SF36 responses were returned from 31 patients with average followup time 35.6 months. On the SF-36 scales in their study, physical and social functioning, role limitations due to physical problems and vitality were limited compared with the average reported for the Dutch population.

We included the instrument LiSat-11, with questions on satisfaction with life as a whole and 10 different items as a way to add information in fields where SF-36 might be weak. Considering the frequent associated injuries to genito-urinary structures it seemed important to include an instrument that includes for instance sexual life. In a retrospective follow-up study by Anke and Fugl-Meyer ${ }^{1}$ in a rehabilitation hospital, a series of 69 patients were seen three years after a multiple trauma. A total of $87 \%$ experienced a decrease in at least one of the life satisfaction items when compared with their life prior to the injury. Significantly fewer patients reported to be satisfied with life as a whole, as well as the domains sexual life, ADL, contact with friends, leisure, vocational and financial situation. Satisfaction with family life and partner relationship did not decrease significantly. This is in accordance with the findings of our study. In a study by Snekkevik et al. ${ }^{18} 26$ patients who had sustained severe multiple 
trauma, without neuropsychological deficits, were prospectively followed for 1-3 years. In conclusion, their patients described that global life satisfaction was considerably reduced at the time of final follow-up when compared with before the trauma (as reported at admission).

In conclusion, pelvic fracture patients reported substantially lower QoL when compared with a reference population, even at two years after their injury and despite good radiological results. As the lower QoL might be attributable not only to the pelvic injury per se, but also to associated injuries, it seems important to involve other specialities during the course of rehabilitation to optimise chances for these severely injured patients to regain their prefracture quality of life.

\section{Conflict of interest}

The authors disclose that there are no conflicts of interest concerning financial or personal relationships with other people, or organisations, that could inappropriately influence their work.

\section{References}

1. Anke AG, Fugl-Meyer AR. Life satisfaction several years after severe multiple trauma-a retrospective investigation. Clin Rehabil 2003;17:431-42.

2. Bryceland JK, Keating JF. Laparotomy and unstable pelvic fractures. Injury 2008;39:853-7.

3. Campbell A, Converse PE, Rodgers WL. The quality of American life: perceptions, evaluations and satisfactions. New York: Russel Sage Foundation; 1976.

4. Failinger MS, McGanity PL. Unstable fractures of the pelvic ring. J Bone Joint Surg Am 1992;74:781-91.

5. Fugl-Meyer AR, Bränholm I-B, Fugl-Meyer KS. Happiness and domain-specific life satisfaction in adult northern Swedes. Clin Rehab 1991;5:25-33.

6. Gribnau AJ, van Hensbroek PB, Haverlag R, et al. U-shaped sacral fractures: surgical treatment and quality of life. Injury 2009;40:1040-8.
7. Harvie P, Chesser TJ, Ward AJ. The Bristol regional pelvic and acetabular fracture service: workload implications of managing the polytraumatised patient. Injury 2008;39:839-43.

8. Katsoulis E, Giannoudis PV. Impact of timing of pelvic fixation on functional outcome. Injury 2006;37:1133-42.

9. Korovessis P, Baikousis A, Stamatakis M, Katonis P. Medium- and long-term results of open reduction and internal fixation for unstable pelvic ring fractures. Orthopedics 2000;23:1165-71.

10. Majeed SA. Grading the outcome of pelvic fractures. J Bone Joint Surg $\mathrm{Br}$ 1989;71:304-6.

11. Marsh JL, Slongo TF, Agel J, et al. Fracture and dislocation classification compendium-2007: Orthopaedic Trauma Association classification, database and outcomes committee. J Orthop Trauma 2007;21:S1-133.

12. Matta JM, Tornetta 3rd P. Internal fixation of unstable pelvic ring injuries. Clin Orthop 1996;129-40.

13. Michaels AJ, Madey SM, Krieg JC, Long WB. Traditional injury scoring underestimates the relative consequences of orthopedic injury. J Trauma 2001;50:389-95. discussion 96.

14. Oliver CW, Twaddle B, Agel J, Routt Jr ML. Outcome after pelvic ring fractures: evaluation using the medical outcomes short form SF-36. Injury 1996;27:63541.

15. Pohlemann T, Gansslen A, Schellwald O, et al. Outcome evaluation after unstable injuries of the pelvic ring. Unfallchirurg 1996;99:249-59.

16. Ponsford J, Hill B, Karamitsios M, Bahar-Fuchs A. Factors influencing outcome after orthopedic trauma. J Trauma 2008;64:1001-9.

17. Schweitzer D, Zylberberg A, Cordova M, Gonzalez J. Closed reduction and iliosacral percutaneous fixation of unstable pelvic ring fractures. Injury 2008;39:869-74.

18. Snekkevik H, Anke AG, Stanghelle JK, Fugl-Meyer AR. Is sense of coherence stable after multiple trauma? Clin Rehabil 2003;17:443-53.

19. Sullivan M, Karlsson J, Ware Jr JE. The Swedish SF-36 Health Survey-I. Evaluation of data quality, scaling assumptions, reliability and construct validity across general populations in Sweden. Soc Sci Med 1995;41:1349-58.

20. Van den Bosch EW, Van der Kleyn R, Hogervorst M, Van Vugt AB. Functional outcome of internal fixation for pelvic ring fractures. J Trauma 1999;47:36571.

21. Ware JE, Kosinski M, Keller SD. SF-36 physical and mental health summary scales: a user's manual. Boston: The Health Institute, New England Medical Center; 1994.

22. White CE, Hsu JR, Holcomb JB. Haemodynamically unstable pelvic fractures. Injury 2009;40:1023-30. 